\title{
Molecular and Phylogenetic Analysis of the Partial Tams1 Gene Sequence of a Vaccine Strain of Theileria annulata
}

\author{
Majid Esmaelizad $^{1 *}$, Saber Jelokhani Niaraki ${ }^{1}$ and Reza Hashemi Fesharaki ${ }^{2}$ \\ ${ }^{1}$ Departments of Biotechnology. ${ }^{2}$ Protozoology; Razi Vaccine \& Serum Research Institute; Karaj - Iran
}

\begin{abstract}
The polypeptide Tams1 is an immunodominant major merozoite piroplasm surface antigen of the protozoan parasite Theileria annulata and is highly variable. In this study, the partial nucleotide (nt) sequence of the Tams1 (522 nt) gene of Iranian vaccine strain (Vaccine-ir68) recovered from an outbreak of disease in Iran was determined and compared with the corresponding sequences of eighteen previously published Tams l genes. According to sequencing result, a novel amino acid substitution at the Tams 1 region $(K \rightarrow Q)$ was found exclusively in isolate Vaccine-ir68. Genetic distance values, estimated from the sequence data, revealed striking sequence homology (approximately 99\%) between Vaccine-ir68 isolate and Tunisian isolates, showing that they were same isolates of T. annulata which were spread in these areas. The phylogenetic tree constructed based on the sequence alignment of 19 Tams 1 coding regions was distinctly divided into five lineages. There might be some unknown tick carrier birds immigrating to the different geographical regions. These birds have an effective role to distribute the T. annulata species in North Africa, Palestine and Iran.
\end{abstract}

Key words: Tams 1 , Theileria Annulata, merozoite surface antigen, phylogenetic

\section{INTRODUCTION}

Tropical theileriosis is a debilitating disease of cattle caused by the protozoan parasite Theileria annulata. The parasite is transmitted by the ticks of the genus Hyalomma which exist in many countries across the northern Africa and southern Europe, extending through the Middle East, India, and southern Russia to China (Jensen et al., 2008; Kotti et al., 2001). The disease has a wide geographical distribution and is endemic in the Mediterranean basin. Theileriosis is the most economically important cattle disease in Iran, causing major losses in livestock production. It is a serious constraint to the cattle breeding programs designed to increase the milk and meat production in the Iran (Hashemi-Fesharki 1990). The mortality rate varies from $90 \%$ in introduced exotic breeds to $5 \%$ or less in indigenous breeds (Neitz 1957). It has been reported that the rate of mortality in Iranian pure and crossbred cattle reached 40 and $80 \%$, respectively (HashemiFesharki 1988).

Tams1, a well-characterised antigen of $T$. annulata, is a member of the major merozoite piroplasm surface antigen (mMPSA) family of polypeptides that have been identified on all the Theileria species studied to-date (Katzer et al., 2002). Tamsl coding region encodes the Tamsl polypeptide with a variable molecular mass of between 30 to $32 \mathrm{kDa}$ (Katzer 1998). Generation and selection of divergent antigenic types has

*Author for correspondence: m.esmaelizad@rvsri.ir 
implications for the inclusion of the Tams 1 antigen in a subunit recombinant vaccine or use in the development of a diagnostic ELISA (Gubbels et al., 2000). d'Oliveira reported the use of PCR for sensitive and specific amplification of $T$. annulata DNA from blood samples obtained from carrier cattle in 1995 (Shiels et al., 1994; d'Oliveira et al., 1995). The advent of PCR technology followed by sequencing has greatly allowed the development of sensitive diagnostic assays and phylogenetic studies for T. annulata (Bishop 1992).

The present work was undertaken with an objective of studying the genetic relationship of Iranian vaccine strain (Vaccine-ir68) with other isolates from different parts of the world, based upon the incomplete sequence of Tams1 gene. In addition, molecular analysis of partial Tamsl region of Vaccine-ir68 isolate was also performed.

\section{MATERIAL AND METHODS}

\section{Organism isolation and vaccine preparation}

A virulent strain of $T$. annulata isolated locally from an outbreak in Iran-Tehran during 1968 was used to prepare an appropriate vaccine. The culture medium was made up of a mixture of Eagle's balanced salt solution and yeast extract lactalbuminhydrolysate (YLH; 20\%). Schizontinfected lymphoid cells, obtained originally from the prescapular gland of an infected calf by the biopsy technique, were inoculated in the culture medium. These lymphoid cells were then successively subcultured at 4-day intervals in the form of suspension and were kept at $37^{\circ} \mathrm{C}$ in laboratory incubator. In the cultures, almost all of the lymphoid cells were infected with the schizonts. Large Roux flasks containing 80 to 100 $\mathrm{ml}$ of medium and 6 to $7 \mathrm{ml}$ of suspension culture (4.2 to $4.9 * 10^{6}$ Schizont-infected lymphoid cells) were used in order to prepare the anti-theileriosis vaccine. After cells had completed the growth, the material was harvested and concentrated to 1.5$2.0 * 10^{6}$ infected cells. The following step was performed by the addition of Glycerol (10\%) and the final suspension $(15 \mathrm{ml})$ was distributed into small 20-ml vials. The vials were transferred to a freezer maintained at $-70{ }^{\circ} \mathrm{C}$ until required
(Hashemi-Fesharki and Shad-Del, 1973). This strain has been used for the immunization of cattle against T. annulata in Iran, since 1973 (HashemiFesharki 1998).

\section{DNA extraction and polymerase chain reaction (PCR)}

Genomic DNA was extracted from the schizontinfected lymphoid cells by phenol-chloroform method as described previously (Sambrook et al., 1989). PCR was carried out using the specific primers to amplify a 522-bp fragment of the Tams1 gene. DNA prepared as described above was amplified in $50 \mu \mathrm{l}$ of reaction mixture containing $20 \mu \mathrm{l}$ of $10 \mathrm{x}$ reaction buffer, $5 \mu \mathrm{l}$ of mixed dNTPs (2.5 mM each), 0.5 unit of Taq DNA polymerase enzyme (Roche Diagnostic, Germany), $1 \mu \mathrm{l}$ of each primer (10 pmol each) designed with DNASIS software: forward (5'-CAT AGA ATT CTT TGA GAT GTT GTC C-3') and reverse (5'-GAT ATC TAG ATA CGA ACA TGG GTT-3'), $4 \mu \mathrm{l}$ of DNA template (100ng), $3 \mu \mathrm{l}$ $25 \mathrm{mM} \mathrm{MgCl}{ }_{2}$ (1.5mM final concentration), and up to $50 \mu \mathrm{l}$ with $\mathrm{ddH}_{2} \mathrm{O}$. The fragments were amplified using a PCR program of: $94^{\circ} \mathrm{C}$ for 3 min, 30 cycles of $\left(94^{\circ} \mathrm{C}\right.$ for $30 \mathrm{~s}, 52^{\circ} \mathrm{C}$ for $30 \mathrm{~s}$, $72^{\circ} \mathrm{C}$ for $\left.40 \mathrm{~s}\right), 72^{\circ} \mathrm{C}$ for $5 \mathrm{~min}$.

\section{Cloning of PCR product, DNA sequencing and data analysis}

The PCR amplicons were analyzed on $1 \%$ agarose gel to check for the correct fragment size. In this regards, amplicons were visualised with ethidium bromide and subsequently extracted and purified from the agarose gel by using the gel extraction kit (Roche). The DNA fragments amplified by PCR were cloned into pTZ57R/T vector (Fermentas, Germany) using standard protocols. DNA sequencing was carried out using $\mathrm{T} 7$ promoter primer in both directions in MWG Co, Germany. Percent identity was made by using the MegAlign project of DNAStar software package (version 5.1). Multiple sequence alignments were analyzed using the BioEdit, version 7 (Hall 1999). Table 1 shows the description of $T$. annulata isolates used in the study. Phylogenetic tree (with bootstrap values) was created using the CLUSTAL X (2.0) and was shown using the NJPLOT program. 
Table 1 - Description of T. annulata field isolates used to determine the sequence variability in the Tams 1 coding region.

\begin{tabular}{cccc}
\hline Serial No. & Isolate & Country & Accession No. \\
\hline 1 & Vaccine-ir68 & Iran & AY672541 \\
2 & Vaccine & Iran & EF092914 \\
3 & Boein-zahra/1 & Iran & EF092918 \\
4 & Boein-zahra/2 & Iran & EF092919 \\
5 & Karaj/78 & Iran & EF092915 \\
6 & Ankara & Turkey & XM948626 \\
7 & Ankara & Turkey & TAU22887 \\
8 & na31 & Turkey & AF214908 \\
9 & tp12 & Portugal & AF214828 \\
10 & tb39 & Bahrain & AF214802 \\
11 & tH5 & India & AF214844 \\
12 & $492 \mathrm{e}$ & Tunisia & AF214903 \\
13 & 234 & Tunisia & AF214895 \\
14 & 233 & Tunisia & AF214894 \\
15 & 231 & Tunisia & AF214893 \\
16 & $08 \# 06$ & Iraq & GU130192 \\
17 & $08 \# 03$ & Iraq & GU130189 \\
18 & $08 \# 02$ & Iraq & FJ159695 \\
19 & tI11 & Italy & AF214863
\end{tabular}

\section{RESULTS}

Partial nucleotide sequence coding for Tams1 polyprotein was determined from the cloned fragments of DNA by PCR amplification and sequencing. As shown in Figure 1, the nucleotide and predicted amino acid sequence of Vaccineir68 (currently used as vaccine strain) was aligned with the corresponding sequences of eighteen previously published Tams 1 genes. The number of Tams 1 sequences analyzed (19 isolates) is listed in Table 1. The nucleotide sequence analysis demonstrated significant similarity $(>99 \%$ nucleotide identity) between the Vaccine-ir68 and Tunisian isolates (Fig. 2). The comparison analysis of Tamsl sequences retrieved from the GenBank revealed a nucleotide exchange at position 376 of the Vaccine-ir68 sequence. Figure 1a showed that the nucleotide 376 was changed from $\mathrm{A}$ to $\mathrm{C}$, resulting in a change in amino acid 126 from Lysine to Glutamine (see Fig. 1b). In contrast to Vaccine-ir68, the remaining eighteen isolates showed no corresponding changes in the same position. Figure 3 shows a phylogenetic tree constructed based on the sequence alignment of 19 gene sequences. The phylogenetic clustering in the Tams1 coding region of all the nineteen field isolates included in the analysis was distinctly divided into five lineages (A-E). The isolate Vaccine-ir68 was clustered with two Iranian and four Tunisian isolates into a separate branch from other T. annulata isolates (lineage C). Two Turkish isolates and lone isolate tp12 were grouped closely together in lineage D. The resulting phylogenetic tree indicated that four isolates (tb39 and tH5) and (tI11 and na31) differed from the Vaccine-ir68 and could be placed in the lineages $\mathrm{E}$ and $\mathrm{A}$, respectively. They showed genetic relationship with 91.6-98.5\% nucleotide identity among themselves and 92.9$94.3 \%$ identity to isolate Vaccine-ir68. Phylogenetic analysis showed that two other Iranian isolates were clustered in the lineage B and are linked to three other isolates from Iraq, 08\#02, $08 \# 03$, and $08 \# 06$, suggesting that they had a close genetic relationship. These isolates might be actually genetically the same isolates of $T$. annulata. The overall topology of the phylogenetic tree presented in Figure 3 suggested that, among all these sequences the Vaccine-ir68 shared the highest homology with the Vaccine isolate and Tunisian isolates (99\% similarity), and the lowest homology with Iraqi and Iranian isolates presented in lineage B (89\% similarity). 
a.

Vaccine-ir68
Vaccine
$08 \# 02$
$08 \# 03$
$08 \# 06$
231
233
234
492 e
Ankara
Ankara-1
Boein Zahra-1
Boein Zahra-2
Karaj-78
na31
tb39
tH5
tI11
tp12

b.

\begin{tabular}{l} 
Vaccine-ir68 \\
\hline Vaccine \\
$08 \# 02$ \\
$08 \# 03$ \\
$08 \# 06$ \\
231 \\
233 \\
234 \\
492 e \\
Ankara \\
Ankara-1 \\
Boein Zahra-1 \\
Boein Zahra-2 \\
Karaj-78 \\
na31 \\
tb39 \\
tH5 \\
tI11 \\
tp12
\end{tabular}

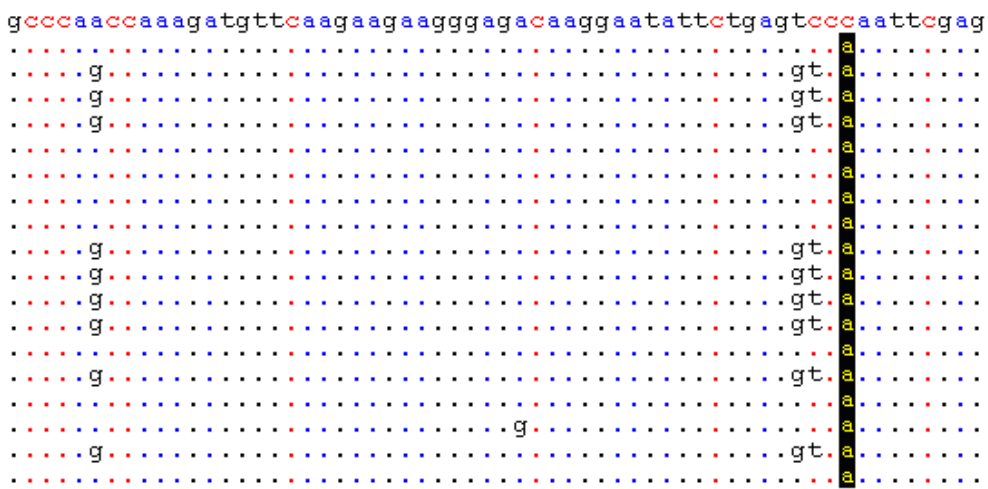

126

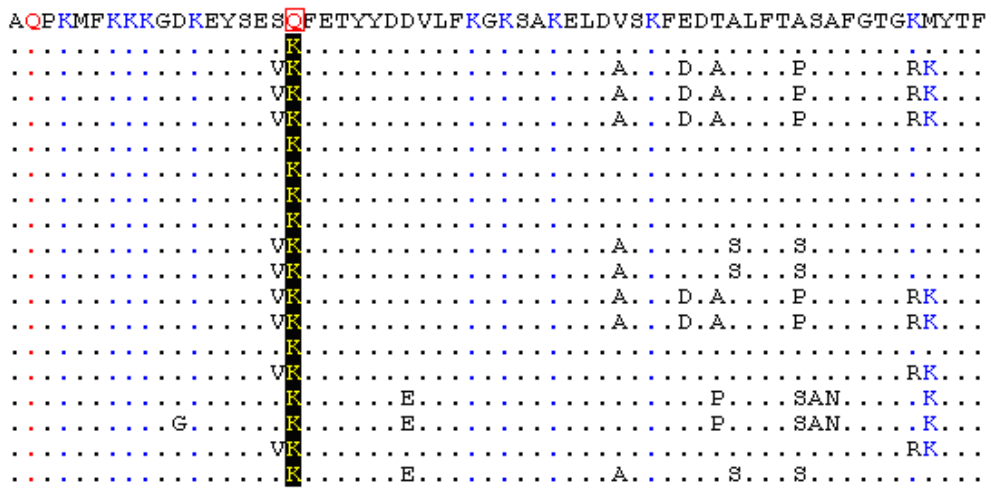

Figure 1 - (a) Alignment of partial nucleotide sequences of Tams1 region. Mutation relevant in this study is highlighted. Dot (.) indicates sequence identity in relation to the Iranian vaccine strain (Vaccine-ir68). (b) The partial amino acid sequence of Tams1 polypeptide of the Iranian vaccine strain (Vaccine-ir68) is shown in the top lane in the one letter code. Aligned to it are sequences of other T. annnulata isolates. Specific residue is marked with a red box. The numbers (126 and 376) above the sequences indicate the positions of substitution from the start of the Tamsl coding region.

\begin{tabular}{|c|c|c|c|c|c|c|c|c|c|c|c|c|c|c|c|c|c|c|c|c|}
\hline \multicolumn{21}{|c|}{ Percent Identity } \\
\hline & 1 & 2 & 3 & 4 & 5 & 6 & 7 & 8 & 9 & 10 & 11 & 12 & 13 & 14 & 15 & 16 & 17 & 18 & 19 & \\
\hline 1 & & 99.8 & 89.3 & 89.3 & 90.4 & 99.6 & 99.6 & 99.6 & 99.6 & 95.4 & 95.4 & 90.8 & 90.8 & 98.4 & 94.3 & 93.7 & 92.9 & 94.1 & 97.1 & 1 \\
\hline 2 & 0.2 & & 89.9 & 89.9 & 90.8 & 99.8 & 99.8 & 99.8 & 99.8 & 95.5 & 95.5 & 91.2 & 91.2 & 98.6 & 94.3 & 93.5 & 92.6 & 94.1 & 97.1 & 2 \\
\hline 3 & 10.9 & 10.5 & & 100.0 & 100.0 & 89.9 & 89.9 & 89.9 & 89.9 & 92.7 & 92.7 & 100.0 & 100.0 & 89.3 & 94.5 & 90.2 & 91.7 & 94.2 & 91.1 & 3 \\
\hline 4 & 10.9 & 10.5 & 0.0 & & 100.0 & 89.9 & 89.9 & 89.9 & 89.9 & 92.7 & 92.7 & 100.0 & 100.0 & 89.3 & 94.5 & 90.2 & 91.7 & 94.2 & 91.1 & 4 \\
\hline 5 & 9.9 & 9.6 & 0.0 & 0.0 & & 90.8 & 90.8 & 90.8 & 90.6 & 92.5 & 92.5 & 100.0 & 100.0 & 89.9 & 95.9 & 89.5 & 92.9 & 94.6 & 91.9 & 5 \\
\hline 6 & 0.4 & 0.2 & 10.5 & 10.5 & 9.6 & & 100.0 & 100.0 & 99.6 & 96.0 & 96.0 & 91.2 & 91.2 & 98.8 & 94.6 & 94.1 & 93.3 & 94.6 & 97.1 & 6 \\
\hline 7 & 0.4 & 0.2 & 10.5 & 10.5 & 9.6 & 0.0 & & 100.0 & 99.6 & 96.0 & 96.0 & 91.2 & 91.2 & 98.8 & 94.6 & 94.1 & 93.3 & 94.6 & 97.1 & 7 \\
\hline 8 & 0.4 & 0.2 & 10.5 & 10.5 & 9.6 & 0.0 & 0.0 & & 99.6 & 96.0 & 96.0 & 91.2 & 91.2 & 98.8 & 94.6 & 94.1 & 93.3 & 94.6 & 97.1 & 8 \\
\hline 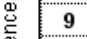 & 0.4 & 0.2 & 10.5 & 10.5 & 9.9 & 0.4 & 0.4 & 0.4 & & 96.0 & 96.0 & 91.0 & 91.0 & 98.8 & 94.4 & 94.1 & 92.9 & 94.3 & 97.1 & 9 \\
\hline 10 & 4.6 & 4.7 & 7.1 & 7.1 & 7.4 & 4.2 & 4.2 & 4.2 & 4.2 & & 100.0 & 92.9 & 92.8 & 96.5 & 92.9 & 93.1 & 91.9 & 92.7 & 97.3 & 10 \\
\hline 11 & 4.5 & 4.7 & 7.1 & 7.1 & 7.4 & 4.1 & 4.1 & 4.1 & 4.1 & 0.0 & & 92.9 & 92.8 & 96.5 & 92.9 & 93.1 & 92.0 & 92.7 & 97.3 & 11 \\
\hline 12 & 9.3 & 9.2 & 0.0 & 0.0 & 0.0 & 9.1 & 9.1 & 9.1 & 9.3 & 7.1 & 7.1 & & 100.0 & 90.4 & 96.1 & 90.0 & 93.3 & 94.9 & 92.3 & 12 \\
\hline 13 & 9.4 & 9.2 & 0.0 & 0.0 & 0.0 & 9.2 & 9.2 & 9.2 & 9.4 & 7.1 & 7.1 & 0.0 & & 90.4 & 96.1 & 90.0 & 93.3 & 94.9 & 92.2 & 13 \\
\hline 14 & 1.6 & 1.4 & 10.9 & 10.9 & 10.3 & 1.2 & 1.2 & 1.2 & 1.2 & 3.6 & 3.6 & 9.8 & 9.9 & & 93.5 & 94.1 & 92.5 & 93.3 & 95.7 & 14 \\
\hline 15 & 5.6 & 5.7 & 5.7 & 5.7 & 4.2 & 5.4 & 5.4 & 5.4 & 5.6 & 7.1 & 7.0 & 4.0 & 4.0 & 6.4 & & 91.8 & 94.8 & 98.5 & 93.1 & 15 \\
\hline 16 & 6.0 & 6.2 & 9.8 & 9.8 & 10.3 & 5.6 & 5.6 & 5.6 & 5.6 & 6.8 & 6.8 & 9.8 & 9.8 & 5.5 & 7.9 & & 96.0 & 91.6 & 92.7 & 16 \\
\hline 17 & 6.8 & 7.1 & 8.1 & 8.1 & 7.0 & 6.4 & 6.4 & 6.4 & 6.8 & 7.9 & 7.9 & 6.6 & 6.6 & 7.1 & 5.0 & 3.7 & & 94.4 & 92.3 & 17 \\
\hline 18 & 5.8 & 6.0 & 6.1 & 6.1 & 5.6 & 5.4 & 5.4 & 5.4 & 5.8 & 7.3 & 7.3 & 5.3 & 5.3 & 6.6 & 1.5 & 8.1 & 5.4 & & 92.5 & 18 \\
\hline 19 & 2.9 & 2.9 & 9.1 & 9.1 & 8.4 & 2.9 & 2.9 & 2.9 & 2.9 & 2.7 & 2.7 & 8.0 & 8.0 & 4.4 & 7.0 & 7.0 & 7.5 & 7.7 & & 19 \\
\hline & 1 & 2 & 3 & 4 & 5 & 6 & 7 & 8 & 9 & 10 & 11 & 12 & 13 & 14 & 15 & 16 & 17 & 18 & 19 & \\
\hline
\end{tabular}

\begin{tabular}{l} 
Vaccine-ir68 \\
\hline Vaccine \\
$08 \# 02$ \\
$08 \# 03$ \\
$08 \# 06$ \\
231 \\
233 \\
234 \\
$492 \mathrm{e}$ \\
Ankara-1 \\
Ankara \\
Boein-zahra-1 \\
Boein-zahra-2 \\
Karaj-78 \\
na31 \\
tb39 \\
tH5 \\
t11 \\
tp12
\end{tabular}

Figure 2 - Percent identity and divergence between Vaccine-ir68 isolate and eighteen published sequences of Tamsl gene. 


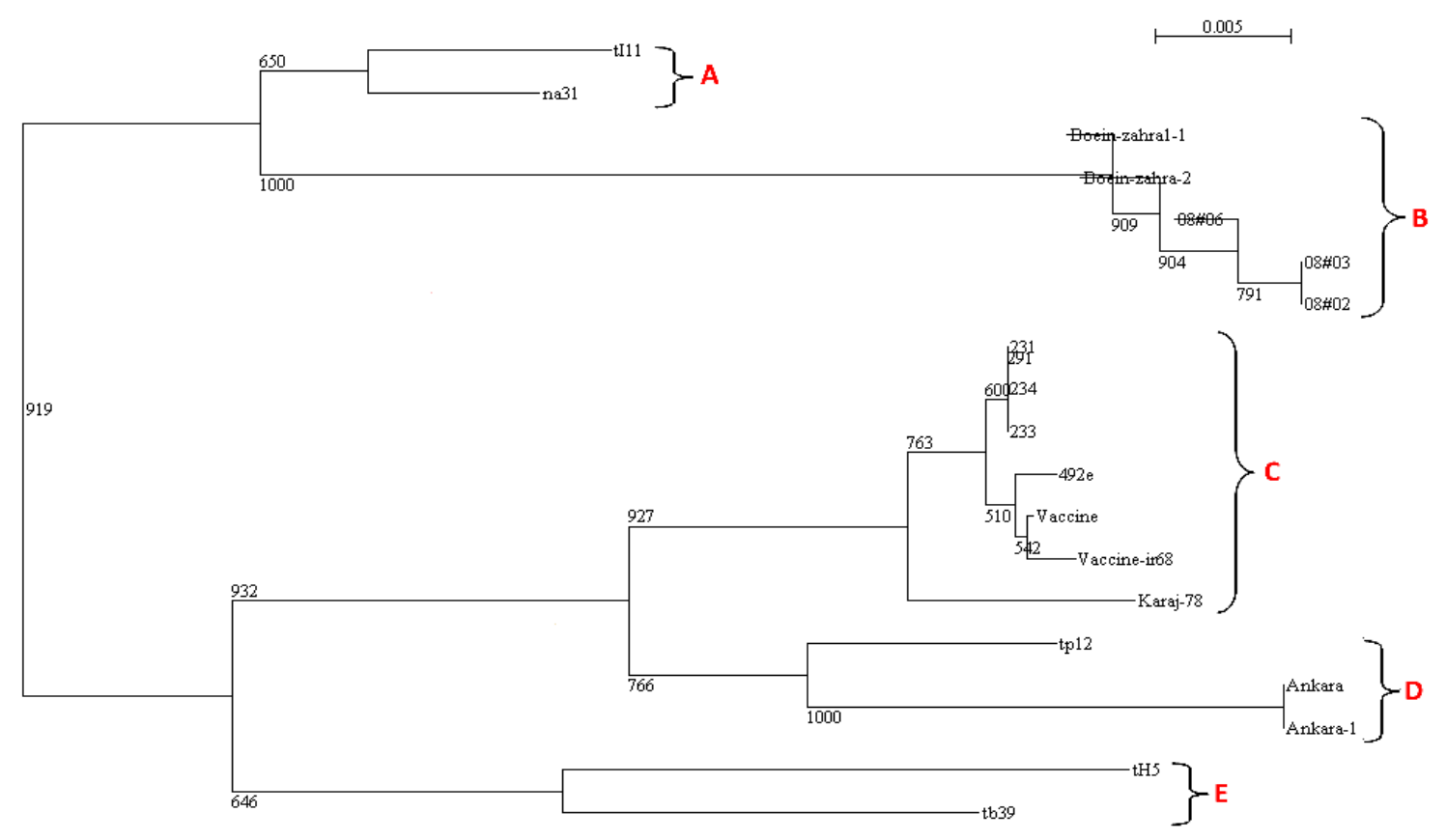

Figure 3 -Phylogenetic tree based on a comparison of Vaccine-ir68 isolate to other T. annulata field isolates, established with Tamsl coding sequences (522 bp). The lineages and bootstrap values are shown on the tree.

\section{DISCUSSION}

Of the few polypeptides that have been located to the merozoite/piroplasm stages of $T$. annulata, the major merozoite piroplasm surface antigen (mMPSA) Tams 1 has been characterized in great detail. The function of Tams 1 and other mMPSA polypeptides have not been defined conclusively. Tams 1 appears to be coded by a single copy gene in the haploid genome of the parasite (Gubbels et al., 2000). The partial nucleotide sequence of the Tams1 (522 nt) gene of Iranian vaccine strain (Vaccine-ir68) enabled us to perform a phylogenetic analysis using Tams1 sequences, originated from various geographical locations, available in the Genbank database. Figure 2 indicates a high level of sequence variability among the isolates. The high level of genetic diversity within the Tamsl polyprotein has been confirmed by the phylogenetic analysis. Gubbels reported that the analysis of the sequences provided evidence for the generation of sequence diversity through the intragenic recombination. It seemed that Tams 1 polymorphism evolved by the selection of amino acid substitutions in the particular regions and exchange of these regions between the alleles during the sexual recombination. Advent of diversity through the positive selection of amino acid substitutions and intragenic recombination have been reported for a number of viral, bacterial, rickettsial and protozoan antigen genes (Gubbels et al., 2000; Katzer et al., 1998). Tamsl sequences and mMPSA sequences from other classical Theileria species reveal the regions where amino acids appear to be conserved. It is believed that the conserved regions play a functional/structural role, while the variable regions are exposed to the immune response (Gubbels et al., 2000). Tams1 gene encodes a protein of 281 amino acids (aa) containing a putative hydrophobic N-terminal signal peptide, which is highly variable (d'Oliveira et al., 1996; Gubbels et al., 2000).

Partial sequences of the Tamsl gene were compared with the Iranian strain (Vaccine-ir68) to estimate the extent of Tams 1 diversity throughout the regions included in this analysis. The deduced amino acid sequences investigated here were variable when compared with each other. The number of sequence differences exhibited by each of the isolates revealed that the Vaccine-ir68 contains amino acid substitution at position 126 of 
the amino acid sequence. This isolate was characterized by a mutation at nucleotide position 376, resulting in a Lys-to-Gln substitution (Fig. 1a). An interesting observation was that in relation to the Vaccine-ir68 isolate the entire isolates revealed no such change. Polymorphism in Tams 1 may be associated with the evasion of the bovine immune system and possibly balancing selection (Weir et al., 2007). Another possible explanation for the high levels of genetic diversity found among the isolates of $T$. annulata could be random mutation of nucleotides during asexual reproduction (Gubbels et al., 2000).

In this work, the similarities of Tunisian and Iranian isolates of $T$. annulata were investigated. The resulting homology showed that they were analogous and closely resembled each other. Information on the prevalence of tick-borne pathogens in the potential vector ticks of the region is essential for the epidemiology of tickborne diseases (Ica et al., 2007). According to the sequence similarity observed among the isolates of Iran, Tunisia and Turkey, and also the location of Palestine as a land bridge between the Africa and Asia, it could be inferred that some unknown immigrant birds might have an effective role to distribute the ticks of the $T$. annulata species from the north Africa to Palestine and then to Turkey, Russia and Iran. In support of this hypothesis, there have been many studies on the wild birds indicating that the wild birds could carry different ectoparasites such as species of Hyalomma ticks (Calvete et al., 2003; Grigor'ev et al., 2001; Kotti et al., 2001; Millan et al., 2004; Mumcuoglu et al., 2005). The sequence analysis based on the Tams 1 gene is in complete agreement with a previous report documenting that there is divergence between $T$. annulata vaccine strains and other available isolates in Iran (Habibi et al., 2007). The mentioned isolates of $T$. annulata sharing $99 \%$ similarity are economically valuable for the vaccine manufacturing industry in order to make the vaccines more affordable for use in developing countries such as Iran, Tunisia and Algeria. The extensive diversity of Tams 1 sequences may make this antigen a difficult candidate for the vaccine production. The attenuated strain of $T$. annnulata in Iran, which is now applied routinely in the vaccine development against the bovine tropical theileriosis is local one and has been originally obtained from an infected calf from Vasfenard village, located in south of Tehran, Iran. In order to consider the Iranian vaccine strain as an appropriate vaccine, all necessary quality and quantity control methods have been implemented. As a result of this project, the vaccine has proved to be effective in prevention of infection in cattle without any clinically significant abnormality and adverse side effects. The duration of vaccineinduced immunity lasted more than one year. Furthermore, different strains with diversity of virulence and clinical symptoms they induced in infected animals (parasitical and thermal reactions) were isolated in Iran from infected cattle. Studies done on inoculating the Algerian $T$. annulata vaccine (Schizont infected blood named Cuba vaccine) into Iranian cattle indicated that Algerian vaccine induced protective immunity against the Iranian strain of $T$.annnulata. The protective immunity induced by the Algerian vaccine was perhaps the decisive reason showing the cross immunity between the strains of Iran and Algeria (Hashemi-Fesharki et al., 1988). The existence of functional motifs conserved across Tams 1 polypeptides must be taken into consideration in the design and manufacture of vaccine, if they are found to be associated with the immune protection. Therefore, all different immunogenic forms of Tamsl antigen should be noted in the vaccine production trends in respect of their biological characteristics.

\section{ACKNOWLEDGEMENTS}

We would like to thank Professor Jabbar Ahmed, ICTTD (Integrated Consortium on Ticks and TickBorne Diseases, Germany) for his continuous advices and kindly comments. This work was financially supported by Razi Vaccine and Serum Research Institute.

\section{REFERENCES}

Bishop, R.; Sohanpal, B.; Kariuki, D. P.; Young, A. S.; Nene, V.; Baylis, H.; Allsopp, B. A.; Spooner, P. R.; Dolan, T. T.; Morzaria, S. P. (1992), Detection of a carrier state in Theileria parva-infected cattle by the polymerase chain reaction. Parasitology. 104, 215232.

Calvete, C.; Estrada, R.; Lucientes, J.; Estrada, A. (2003), Ectoparasite ticks and chewing lice of redlegged partridge, Alectoris rufa, in Spain. Med Vet Entomol. 17, 33-37. 
d'Oliveira, C.; Tijhaar, E. J.; Shiels, B. R.; Van Der Weide, M.; Jongejan, F. (1996), Expression of genes encoding two major Theileria annulata merozoite surface antigens in Escherichia coli and a Salmonella typhimurium aroA vaccine strain. Gene. 172, 33-39

d'Oliveira, C.; Van Der Weide, M.; Habela, M. A. (1995), Detection of Theileria annulata in blood samples of carrier cattle by PCR. J Clin Microbiol. 33, 2665-2669.

Grigor'ev, M. P.; Evchenko, I. U. M.; Shaposhnikova, L. I.; Shchenetts, K. V.; Emel'ianov, S. A.; Ermolova, N. V.; Tikhenko, N. I.; Levchenko, B. I. (2001), Role of some wild birds and mammals in the natural foci of Crimean haemorrhagic fever in Stavropol' region. Zh Mikrob Epid Immun. 6, 92-95.

Gubbels, M. J.; d'Oliveira, C.; Jongejan, F. (2000), Development of an Indirect Tams1 Enzyme-Linked Immunosorbent Assay for Diagnosis of Theileria annulata Infection in Cattle. Clin Diag Lab Immunol. 7, 404-411.

Gubbels, M. J.; Katzer, F.; Hide, G.; Jongejan, F.; Shiels, B. R. (2000), Generation of a mosaic pattern of diversity in the major merozoite-piroplasm surface antigen of Theileria annulata. Mol Biochem Parasit. 110, 23-32.

Habibi, G. R.; Esmaeil-Nia, K.; Bozorgi, S.; Najjar, E.; Hashemi-Fesharki, R.; Bordbar, N. (2007), PCRbased detection of Theileria infection and molecular characterization of Tams1 T. annulata vaccine strain. Arch Razi Inst. 62, 83-89.

Hall, T. A. (1999), BioEdit: a user-friendly biological sequence alignment editor and analysis, program for Windows 95/98/NT. Nucl Acids Symp. 41, 95-98.

Hashemi-Fesharki, R. (1988), Control of Theileria annulata in Iran. Parasitol Today. 4, 36-40.

Hashemi-Fesharki, R. (1998), Recent development in control of Theileria annulata in Iran. Parasite. 5, 193-196.

Hashemi-Fesharki, R.; Shad-Del, F. (1973), Vaccination of calves and milking cows with different strains of Theileria annulata. Amer $J$ Vet Res. 34, 1465-1467.

Hashemi-Fesharki, R. (1990), Theileriosis due to Theileria annulata in Iran: Recent developments in the research and control of Theileria annulata. Proceeding of a workshop. 17-19 september, Nairobi, Kenya.

Ica, A.; Vatansever, Z.; Yildirim, A.; Duzlu, O.; Inci, A. (2007), Detection of Theileria and Babesia species in ticks collected from cattle. Vet Parasitol. 148, 156160 .
Jensen, K.; Paxton, E.; Waddington, D.; Talbot, R.; Darghouth, M. A.; Glass, E. J. (2008), Differences in the transcriptional responses induced by Theileria annulata infection in bovine monocytes derived from resistant and susceptible cattle breeds. Int J Parasitol. 38, 313-325.

Katzer, F.; McKellar, S.; Ben Miled, L.; d'Oliveira, C.; Shiels, B. (1998), Selection for antigenic diversity of Tams1, the major merozoite antigen of Theileria annulata. Ann N Y Acad Sci. 849, 96-108.

Katzer, F.; McKellar, S.; Ferguson, M. A.; d'Oliveira, C.; Shiels, B. R. (2002), A role for tertiary structure in the generation of antigenic diversity and molecular association of the Tams1 polypeptide in Theileria annulata. Mol Biochem Parasitol. 122, 55-67.

Kotti, B. K.; Shaposhnikova, L. I.; Evchenko, I. U. M.; Levchenko, B. I.; Surkhaev, D. B.; Korzhov, P. N.; Tokhov, I. U. M. (2001), Hyalomma marginatum Koch in Stavropol' region. Zh Mikrob Epid Immun. 6, 105-108.

Millan, J.; Gortazar, C.; Martin-Mateo, M. P.; Villafuerte, R. (2004), Comparative survey of the ectoparasite fauna of wild and farm-reared red-legged partridges (Alectoris rufa), with an ecological study in wild populations. Parasitol Res. 93, 79-85.

Mumcuoglu, K. Y.; Banet-Noach, C.; Malkinson, M.; Shalom, U.; Galun, R. (2005), Argasid ticks as possible vectors of West Nile virus in Israel. Vector Borne Zoonotic Dis. 5, 65-71.

Neitz, W. O. (1957), Theileriosis, gonderioses and cytauxzoonoses: a review. Onderstepoort. J Vet Res. 27, 275-430.

Sambrook, J.; Fritsch, E. F.; Maniatis, T. (1989), Molecular cloning: A laboratory Manual. 2th ed. Cold Spring Harbor Laboratory Press, Cold Spring Harbor, New York.

Shiels, B. R.; Smyth, A.; Dickson, J.; McKellar, S.; Tetley, L.; Fujisaki, K.; Hutchinson, B.; Kinnaird, J. H. (1994), A stoichiometric model of stage differentiation in the protozoan parasite Theileria annulata. Mol Cell Differ. 2, 101-125.

Weir, W.; Ben-Miled, L.; Karagenc, T.; Katzer, F.; Darghouth, M.; Shiels, B.; Tait, A. (2007), Genetic exchange and sub-structuring in Theileria annulata populations. Mol Biochem Parasit. 154, 170-180.

Received: August 13, 2010; Revised: March 24, 2011; Accepted: August 16, 2011. 


\section{PÁGINA EM BRANCO}

